\title{
ON THE MODULI SPACE OF CLASSICAL DYNAMICAL R-MATRICES
}

\author{
Pavel Etingof and Olivier Schiffmann
}

\section{Introduction}

A classical dynamical r-matrix is an l-equivariant function $r: \mathfrak{r}^{*} \rightarrow \mathfrak{g} \otimes \mathfrak{g}$ (where $\mathfrak{l}, \mathfrak{g}$ are Lie algebras), such that $r^{21}+r=\Omega$ is $\mathfrak{g}$-invariant, which satisfies the classical dynamical Yang-Baxter equation (CDYBE). CDYBE is a differential equation, which generalizes the usual classical Yang-Baxter equation. It was introduced in 1994 by G.Felder [Fe], in the context of conformal field theory. Solutions of CDYBE and their quantizations appear naturally in several mathematical theories: the theory of integrable systems, special functions, representation theory (see [ES] for a review).

Since classical dynamical r-matrices were introduced, several authors tried to study and classify them $([\mathrm{EV}],[\mathrm{S}],[\mathrm{Xu}])$. The goal of this paper is to describe the moduli space of classical dynamical r-matrices modulo gauge transformations. In particular, we improve and generalize the results of [EV], [S], as well as correct some errors that occurred in these papers (See remarks 3 and 5).

The main achievement of this paper, compared to the previous ones, is that its results are valid for dynamical $r$-matrices for a nonabelian Lie algebra $\mathfrak{l}$. It turns out that this generalization not only brings in new interesting examples (see $[\mathrm{EV}],[\mathrm{AM}]$ ) but also makes the general theory much more clear and natural.

The composition of the paper is as follows.

In Section 1, we recall the definition of a dynamical r-matrix.

In Section 2, we extend to the nonabelian case the notion of a gauge transformation of dynamical r-matrices, introduced in $[\mathrm{EV}]$.

In Section 3, we decribe the space of dynamical r-matrices modulo gauge transformations (the moduli space). Here we formulate our main theorem, stating that under some technical conditions, the moduli space can be identified with a certain explicitly given affine variety. For instance, if $\mathfrak{l}=\mathfrak{g}$, this variety consists of one point, which is the Alekseev-Meinrenken solution [AM] (for semisimple Lie algebras, it was also constructed in [EV]).

In Section 4 we prove the main theorem.

In the appendix, we construct a generalization of the Alekseev-Meinrenken classical dynamical r-matrix, associated to any finite-dimensional Lie algebra $\mathfrak{g}$

Received June 6, 2000. 
with a nondegenerate invariant form and an automorphism $B$ of $\mathfrak{g}$ of finite order which preserves this form.

We don't treat the case of dynamical r-matrices with spectral parameters here. However, we expect some results similar to our main theorem to hold also in this case.

\section{The dynamical Yang-Baxter equation}

Let $\mathfrak{g}$ be a Lie algebra over $\mathbb{C}$, and $\mathfrak{l} \subset \mathfrak{g}$ a finite dimensional Lie subalgebra. Let $x_{1}, \ldots, x_{r}$ be a basis of $\mathfrak{l}$.

Let $D \subset \mathfrak{l}^{*}$ be the formal neighborhood of 0 . Let $V$ be a complex vector space. By functions from $D$ to $V$ we will mean elements of the space $V\left[\left[x_{1}, \ldots, x_{r}\right]\right]$, where we regard $x_{i}$ as coordinates on $D$. Finally, if $\omega \in \Omega^{k}(D, V)$ is a $k$-form with values in any vector space $V$, we denote by $\bar{\omega}: D \rightarrow \Lambda^{k} \mathfrak{l} \otimes V$ the associated function. For an element $r \in \mathfrak{g} \otimes \mathfrak{g}$ we define the classical Yang-Baxter operator

$$
C Y B(r)=\left[r^{12}, r^{13}\right]+\left[r^{12}, r^{23}\right]+\left[r^{13}, r^{23}\right] .
$$

The classical dynamical Yang-Baxter equation (CDYBE) is the following differential equation for an l-equivariant function $r: D \rightarrow \mathfrak{g} \otimes \mathfrak{g}$ :

$$
\operatorname{Alt}(\overline{d r})+C Y B(r)=0,
$$

where for $x \in \mathfrak{g}^{\otimes 3}$, we let $\operatorname{Alt}(x)=x^{123}-x^{213}+x^{231}$.

It is useful to consider solutions of CDYBE which satisfy an additional quasiunitarity condition:

$$
r+r^{21}=\Omega \in\left(S^{2} \mathfrak{g}\right)^{\mathfrak{g}} .
$$

It is easy to show that if $r$ satisfies CDYBE and the quasi-unitarity condition then $\Omega$ is a constant function of $\lambda$.

An l-equivariant solution of CDYBE which satisfies the quasi-unitarity condition is called a dynamical r-matrix. The set of all dynamical r-matrices satisfying (2.2) will be denoted by $\operatorname{Dynr}(\mathfrak{g}, \mathfrak{l}, \Omega)$.

Remark. In the litterature (see for instance [ES] and the references therein) dynamical $\mathrm{r}$-matrices are allowed to have a pole at $\lambda=0$. However, if $\mathfrak{l}$ is abelian and $r(\lambda)$ is a dynamical $\mathrm{r}$-matrix then for any $\lambda_{0} \in \mathfrak{l}^{*}, r\left(\lambda+\lambda_{0}\right)$ is also a dynamical r-matrix. Hence, for classification purposes it is always possible to assume that $r(\lambda)$ is regular at the origin.

\section{Gauge transformations}

Here we will reproduce some results from $[\mathrm{EV}]$, but unlike $[\mathrm{EV}]$, we will not assume that $\mathfrak{l}$ is abelian. We will assume, however, that $\mathfrak{g}$ is finite dimensional.

Let $G$ be the simply connected complex Lie group such that $\operatorname{Lie}(G)=\mathfrak{g}$. Let $g: D \rightarrow G$ be any regular, l-equivariant map. Consider the 1 -form $\eta_{g}=g^{-1} d g$ and set $\zeta_{g}=\left[{\overline{\eta_{g}}}^{12},{\overline{\eta_{g}}}^{13}\right]$. Define an l-equivariant function $\tau_{g}: D \rightarrow \Lambda^{2} \mathfrak{g}$ by the 
formula $\tau_{g}(\lambda)=(\lambda \otimes 1 \otimes 1) \zeta_{g}(\lambda)$. For any l-equivariant function $r: D \rightarrow \mathfrak{g} \otimes \mathfrak{g}$ we set

$$
r^{g}=(g \otimes g)\left(r-\overline{\eta_{g}}+{\overline{\eta_{g}}}^{21}+\tau_{g}\right)\left(g^{-1} \otimes g^{-1}\right) .
$$

The following theorem is a nonabelian generalization of Proposition 1.2 of [EV].

Proposition 3.1. The function $r$ is a dynamical r-matrix if and only if the function $r^{g}$ is.

Proof. Let us show that if $r$ is a dynamical r-matrix then so is $r^{g}$. The other direction is analogous. Let $X=(D \times G \times D,\{\}$,$) be the dynamical Poisson$ groupoid associated to $r$ in [EV]. Consider the automorphism $\sigma$ of $X$ given by $\sigma\left(u_{1}, x, u_{2}\right)=\left(u_{1}, g\left(u_{1}\right) x g\left(u_{2}\right)^{-1}, u_{2}\right)$. Then $\sigma$ transforms $\{$,$\} into the Poisson$ bracket $\{f, g\}_{\sigma}=\sigma^{-1}\{\sigma f, \sigma g\}$. It is straightforward to calculate that the corresponding transformation at the level of dynamical $r$-matrices is exactly (3.1).

The transformation $r \rightarrow r^{g}$ is called a gauge transformation. Note that (3.1) defines an action of the $\operatorname{group} \operatorname{Map}(D, G)^{\mathfrak{l}}$ on $\operatorname{Dynr}(\mathfrak{g}, \mathfrak{l}, \Omega)$, i.e we have $\left(r^{g_{1}}\right)^{g_{2}}=r^{g_{2} g_{1}}$ for any $g_{1}, g_{2} \in \operatorname{Map}(D, G)^{\mathfrak{l}}$ and $r \in \operatorname{Dynr}(\mathfrak{g}, \mathfrak{l}, \Omega)$. Let us denote by $\operatorname{Map}_{0}(D, G)^{\mathfrak{l}}$ the subgroup consisting of maps $g$ satisfying $g(0)=1$. We would like to understand the moduli space

$$
\mathcal{M}(\mathfrak{g}, \mathfrak{l}, \Omega)=\operatorname{Dynr}(\mathfrak{g}, \mathfrak{l}, \Omega) / \operatorname{Map}_{0}(D, G)^{\mathfrak{l}} .
$$

In the triangular case (i.e when $\Omega=0$ ) this space was considered by $\mathrm{P}$. Xu in $[\mathrm{Xu}]$.

Remark 1. It is clear that $\operatorname{Map}(D, G)^{\mathfrak{l}} / \operatorname{Map}_{0}(D, G)^{\mathfrak{l}} \simeq G^{\mathfrak{l}}$. Hence the complete moduli space $\overline{\mathcal{M}}(\mathfrak{g}, \mathfrak{l}, \Omega)=\operatorname{Dynr}(\mathfrak{g}, \mathfrak{l}, \Omega) / \operatorname{Map}(D, G)^{\mathfrak{l}}$ is equal to $\mathcal{M}(\mathfrak{g}, \mathfrak{l}, \Omega) / G^{\mathfrak{l}}$ where $g \in G^{\mathrm{l}}$ acts by $r^{g}=\operatorname{Ad}(g \otimes g)(r)$.

\section{The structure of $\mathcal{M}(\mathfrak{g}, \mathfrak{l}, \Omega)$}

From now on we will assume that

i) $\mathfrak{l} \subset \mathfrak{g}$ has an $\mathfrak{l}$-invariant complement $\mathfrak{m}$.

The following theorem is a generalization of Theorem 1.4 in [EV]. It shows that the space of dynamical r-matrices is, up to gauge equivalence, finite dimensional.

Theorem 1. Let $\rho, r: D \rightarrow \mathfrak{g}^{\otimes 2}$ be two dynamical $r$-matrices such that $r(0)=$ $\rho(0)$. Then there exists $g \in \operatorname{Map}(D, G)^{\mathfrak{l}}$ such that $\rho=r^{g}$.

The proof is a generalization of the proof in $[\mathrm{EV}]$. Before giving it we state the following auxiliary result.

Lemma 4.1 (equivariant Poincaré lemma). Let $\mathfrak{l}$ be a finite-dimensional Lie algebra, $V$ a finite-dimensional $\mathfrak{l}$-module, $k \geq 1$ and $\omega \in \Omega^{k}(D, V)$ an $\mathfrak{l}$-equivariant closed $k$-form with values in $V$. Then there exists an l-equivariant $k-1$-form $\zeta \in \Omega^{k-1}(D, V)$ such that $d \zeta=\omega$. 
Proof. The proof is the same as that for the usual Poincaré lemma. It is enough to assume that $\omega$ is homogeneous, of degree $l \in \mathbb{N}$. Let $E=\sum_{i} x_{i} \frac{\partial}{\partial x_{i}}$ be the Euler vector field on $D$. Then by Cartan's homotopy formula,

$$
l \omega=L_{E} \omega=i_{E} d \omega+d i_{E} \omega=d\left(i_{E} \omega\right)
$$

and we can set $\zeta=i_{E} \omega / l$. Note that $E$ is l-equivariant, hence so is $\zeta$.

Proof of Theorem 1. The dynamical r-matrices $r, \rho$ are by definition formal power series in the variables $x_{i}$. Let us assume that the statement of the theorem holds modulo terms of degree $\geq K$. Let $g_{k}: U \rightarrow G$ be a gauge transformation such that $E:=r^{g_{k}}-\rho$ has degree $\geq K$ and let $E_{K}$ be the homogeneous component of $E$ of degree $K$. Then Alt $\left(\overline{d E_{K}}\right)=\left[C Y B\left(r^{g_{K}}\right)-C Y B(\rho)\right]_{K-1}$ where $[\cdot]_{K-1}$ denotes the homogeneous component of degree $K-1$. But $\left[r^{g_{K}}-\rho\right]_{l}=0$ for all $l<K$ by assumption, hence

$$
\operatorname{Alt}\left(\overline{d E_{K}}\right)=0 .
$$

Lemma 4.2. The exists an $\mathfrak{l}$-equivariant closed 1 -form $\zeta \in \Omega^{1}(D, \mathfrak{g})$ such that $E_{K}=\bar{\zeta}^{21}-\bar{\zeta}$.

Proof. Let us write $E_{K}=E_{\mathfrak{l l}}+E_{\mathfrak{l m}}-E_{\mathfrak{l m}}^{21}+E_{\mathfrak{m} \mathfrak{m}}$ where $E_{\mathfrak{l l}} \in \Lambda^{2} \mathfrak{l}, E_{\mathfrak{l m}} \in \mathfrak{l} \otimes \mathfrak{m}$ and $E_{\mathfrak{m m}} \in \Lambda^{2} \mathfrak{m}$. From (4.1) it follows that $d E_{\mathfrak{m} \mathfrak{m}}=0$ hence $E_{\mathfrak{m m}}=0$. Now let $\xi \in \Omega^{1}(D, \mathfrak{m})$ be such that $\bar{\xi}=E_{\mathfrak{l m}}$. Then (4.1) implies that $\xi$ is closed. Note that the assumption i) guarantees that $\xi$ is equivariant. Finally, let $\omega \in \Omega^{2}(D, \mathbb{C})$ be such that $\bar{\omega}=E_{\mathfrak{l}}$. Then (4.1) says that $\omega$ is closed. By the equivariant Poincaré lemma, there exists an equivariant 1-form $\eta$ such that $d \eta=\omega$. Set $\theta=d \bar{\eta}$, so that $\bar{\theta}-\bar{\theta}^{21}=\bar{\omega}$. Then $\zeta=\xi+\theta$ satisfies the conditions of the lemma.

We now conclude the proof of Theorem 1 . Let $\chi: D \rightarrow \mathfrak{g}$ be any l-equivariant function of order $K+1$ such that $d \chi=\zeta$. Set $g=e^{\chi}$. Then $\eta_{g}=g^{-1} d g$ is of order $\geq K$ and $\bar{\zeta}-\overline{\eta_{g}}$ is of order $\geq K+1$. But then $\bar{\zeta}^{21}-\bar{\zeta}-\left({\overline{\eta_{g}}}^{21}-\overline{\eta_{g}}+\tau_{g}\right)$ is also of order $\geq K+1$. Set $g_{K+1}=g g_{K}$. Then, by the above $r^{g_{K+1}}-\rho$ is of degree $\geq K+1$. The proof follows by induction.

Proposition 4.1. Any dynamical $r$-matrix $r$ is gauge-equivalent to a dynamical $r$-matrix $\rho$ such that $\rho(0) \in \frac{\Omega}{2}+\Lambda^{2} \mathfrak{m}$.

Proof. Let $\bar{\eta}_{0} \in \mathfrak{l} \otimes \mathfrak{g}$ such that $r(0)-\bar{\eta}_{0}+\bar{\eta}_{0}^{21} \in \frac{\Omega}{2}+\Lambda^{2} \mathfrak{m}$. Since $\mathfrak{m}$ is l-invariant, we have $\bar{\eta}_{0} \in(\mathfrak{l} \otimes \mathfrak{g})^{\mathfrak{l}}$. By the equivariant Poincaré lemma, there exists an equivariant function $\chi: D \rightarrow \mathfrak{g}$ satisfying $\chi(0)=0, d \chi=\eta_{0}$. Set $g=e^{\chi}$. Then $\rho:=r^{g}$ satisfies the CDYBE and $\rho(0) \in \frac{\Omega}{2}+\Lambda^{2} \mathfrak{m}$.

Consider the following algebraic variety

$$
\mathcal{M}_{\Omega}=\left\{x \in \frac{\Omega}{2}+\left(\Lambda^{2} \mathfrak{m}\right)^{\mathfrak{l}} \mid C Y B(x)=0 \text { in } \Lambda^{3}(\mathfrak{g} / \mathfrak{l})\right\}
$$


It is immediate from (3.1) that if $\rho$ and $r$ are gauge-equivalent and if $\rho(0) \in$ $\frac{\Omega}{2}+\Lambda^{2} \mathfrak{m}$ and $r(0) \in \frac{\Omega}{2}+\Lambda^{2} \mathfrak{m}$ then $r(0)=\rho(0)$. Moreover, it follows from the CDYBE (2.1) that for every dynamical r-matrix $r \in \operatorname{Dynr}(\mathfrak{g}, \mathfrak{l}, \Omega)$ such that $r(0) \in \frac{\Omega}{2}+\Lambda^{2} \mathfrak{m}$ we have $r(0) \in \mathcal{M}_{\Omega}$.

Hence Theorem 1 and Proposition 3.1 give the following corollary.

Corollary 4.1. The map $\mathcal{M}(\mathfrak{g}, \mathfrak{l}, \Omega) \rightarrow \mathcal{M}_{\Omega}$ which sends a class $\mathcal{C}$ to $r(0)$ where $r \in \mathcal{C}$ is any representative such that $r(0) \in \frac{\Omega}{2}+\Lambda^{2} \mathfrak{m}$, is an embedding.

Remark 2. If condition i) fails then the space $\mathcal{M}(\mathfrak{g}, \mathfrak{l}, \Omega)$ may be infinite-dimensional. This is demonstrated by the following example due to $\mathrm{P} . \mathrm{Xu}[\mathrm{Xu}]$. Let $\mathfrak{g}=\mathbb{C} x \oplus \mathbb{C} y$ be the two-dimensional Lie algebra with $[x, y]=y$, and set $\mathfrak{l}=\mathbb{C} y$. Then $\Lambda^{3} \mathfrak{g}=0$ and $\Lambda^{2} \mathfrak{g}$ is a trivial $\mathfrak{l}$-module. Thus any function $r: D \rightarrow \Lambda^{2} \mathfrak{g}$ is a dynamical $r$-matrix. On the other hand, $\mathfrak{g}^{\mathfrak{l}}=\mathfrak{l}$ and all gauge transformations act trivially.

Remark 3. We would like to use this opportunity to correct the statement of Theorem 1.4 of [EV]. This theorem is incorrect as stated (as shown by Cu's counterexample, see Remark 2). The mistake is in the proof of Lemma 1.5, which uses the incorrect statement that

$$
(\mathfrak{g} \otimes \mathfrak{l} \oplus \mathfrak{l} \otimes \mathfrak{g})^{\mathfrak{l}}=\left(\mathfrak{g}^{\mathfrak{l}} \otimes \mathfrak{l} \oplus \mathfrak{l} \otimes \mathfrak{g}^{\mathfrak{l}}\right)^{\mathfrak{l}}
$$

for commutative $\mathfrak{l}$. This statement, however, is correct with the additional assumption i); in this case Theorem 1.4 of $[\mathrm{EV}]$ and its proof are correct, and Theorem 1.4 of $[\mathrm{EV}]$ is a special case of Theorem 1 above.

Now suppose that $\mathfrak{l}=\mathfrak{g}$. Note that $\mathfrak{i}$ ) automatically holds in this case. Then by Proposition 3.1 and Theorem 1 there is at most one gauge-equivalence class of dynamical r-matrices $r: D \rightarrow \frac{\Omega}{2}+\Lambda^{2} \mathfrak{g}$. Such a class in fact always exists, as was discovered by Alekseev and Meinrenken $[\mathrm{AM}]$. A representative of this class is constructed as follows.

Let $\mathfrak{g}_{\Omega}$ be the ideal of $\mathfrak{g}$ spanned by the components of $\Omega$, and let $D_{\Omega}$ be the formal neighborhood of 0 in $\mathfrak{g}_{\Omega}^{*}$. Let us identify $\mathfrak{g}_{\Omega}$ with $\mathfrak{g}_{\Omega}^{*}$ via $\Omega$. Set $f(s)=\frac{1}{s}-\frac{1}{2} \operatorname{cotanh}\left(\frac{s}{2}\right)$. Then $f$ is smooth at the origin. Consider the following map

$$
\begin{aligned}
T: D_{\Omega} & \rightarrow \operatorname{End}(\mathfrak{g}) \simeq \mathfrak{g}^{*} \otimes \mathfrak{g} \simeq \mathfrak{g} \otimes \mathfrak{g} \\
u & \mapsto f(\operatorname{ad} \mu)
\end{aligned}
$$

Let $\pi^{*}: \mathfrak{g}^{*} \rightarrow \mathfrak{g}_{\Omega}^{*}$ be the projection and set

$$
r_{A M}^{\mathfrak{g}}=\frac{\Omega}{2}+T \circ \pi^{*}: D \rightarrow \mathfrak{g} \otimes \mathfrak{g} .
$$

Theorem $2([\mathrm{AM}])$. The map $r_{A M}^{\mathfrak{g}}$ is a dynamical r-matrix.

This theorem is proved in $[\mathrm{AM}]$ in the case of compact Lie algebras, but the proof can be adapted to the general case. Another proof is given in the appendix.

Corollary 4.2. The moduli space $\mathcal{M}(\mathfrak{g}, \mathfrak{g}, \Omega)$ consists of the single class $r_{A M}^{\mathfrak{g}}$. 
Remark 4. When $\mathfrak{g}$ is a simple Lie algebra and $\mathfrak{l}=\mathfrak{g}$ these results easily follow from $[\mathrm{EV}]$, Section 3.8.

We will now show that, under some technical conditions on $\Omega$, the embedding defined in Corollary 3.1 is actually an isomorphism. From now on we assume that

ii) We have $\Omega \in(\mathfrak{l} \otimes \mathfrak{l}) \oplus(\mathfrak{m} \otimes \mathfrak{m})$.

We will write $\Omega_{\mathfrak{l}}\left(\right.$ resp. $\Omega_{\mathfrak{m}}$ ) for the corresponding components of $\Omega$.

Condition ii) is satisfied in particular in the triangular case $(\Omega=0)$. It is also satisfied when $\mathfrak{l}$ acts semisimply on $\mathfrak{g}$ and when the restriction of the inverse form $()=,\Omega^{-1}$ to $\mathfrak{l}_{\Omega}=\mathfrak{l} \cap \mathfrak{g}_{\Omega}$ is nondegenerate. Indeed, let $\mathfrak{g}^{\prime}$ be an $\mathfrak{l}$-invariant complement of $\mathfrak{l}+\mathfrak{g}_{\Omega}$ in $\mathfrak{g}$ and let $\mathfrak{m}_{\Omega}$ be the orthogonal complement of $\mathfrak{l}_{\Omega}$ i $\mathfrak{g}_{\Omega}$. Then $\mathfrak{m}=\mathfrak{g}^{\prime} \oplus \mathfrak{m}_{\Omega}$ satisfies conditions i) and ii).

Proposition 4.2. Any dynamical r-matrix $r$ is gauge-equivalent to a dynamical $r$-matrix of the form $\rho=r_{A M}^{\mathfrak{l}}+\frac{\Omega_{\mathfrak{m}}}{2}+t$ with $t: D \rightarrow \Lambda^{2} \mathfrak{m}$.

Proof. By Proposition 3.1 there exists a dynamical $r$-matrix $\rho_{0}$ gauge-equivalent to $r$ such that $\rho_{0}(0) \in \frac{\Omega}{2}+\Lambda^{2} \mathfrak{m}$. We will first construct a sequence of gauge transformations $g_{i}, i=1, \ldots$ such that $\rho_{0}^{g_{i}} \in \frac{\Omega}{2}+\left(\Lambda^{2} \mathfrak{l} \oplus \Lambda^{2} \mathfrak{m}\right)$ modulo terms of degree $\geq i$. We set $g_{1}=1$. Suppose that we have constructed $g_{i}$ and let $E_{i}$ be the term of degree exactly $i$ of $\rho_{0}^{g_{i}}$. From the CDYBE we have

$$
-\operatorname{Alt}\left(\overline{d E_{i}}\right)=\left[C Y B\left(\rho_{0}^{g_{i}}\right)\right]_{i-1}
$$

where $[\cdot]_{i-1}$ denotes the component of degree $i-1$. But by our assumption we have $\rho_{0}^{g_{i}} \in \frac{\Omega}{2}+\left(\Lambda^{2} \mathfrak{l} \oplus \Lambda^{2} \mathfrak{m}\right)$ in degrees $\leq i-1$. Using the l-invariance of $\mathfrak{m}$ it is easy to see that this implies that

$$
\left[C Y B\left(\rho_{0}^{g_{i}}\right)\right]_{i-1} \in \operatorname{Alt}((\mathfrak{l} \otimes \mathfrak{m} \otimes \mathfrak{m}) \oplus(\mathfrak{l} \otimes \mathfrak{l} \otimes \mathfrak{l}) \oplus(\mathfrak{m} \otimes \mathfrak{m} \otimes \mathfrak{m})) .
$$

Let $\xi \in \Omega^{1}(D, \mathfrak{m})$ such that $E_{i}+\bar{\xi}^{21}-\bar{\xi} \in \Lambda^{2} \mathfrak{l} \oplus \Lambda^{2} \mathfrak{m}$. Then from (4.3) and (4.4) it follows that $d \xi=0$. By the equivariant Poincaré lemma there exists an equivariant map $\chi: D \rightarrow \mathfrak{m}$ such that $\xi=d \chi$. Moreover, $\xi$ is of degree $\geq i$, hence $\chi$ is of degree $\geq i+1$. Now set $g=e^{\chi}$. Then $\eta_{g}-\xi$ is of order $\geq i+1$. Thus

$$
(g \otimes g)\left(\rho_{0}^{g_{i}}+{\overline{\eta_{g}}}^{21}-\overline{\eta_{g}}+\tau_{g}\right)\left(g^{-1} \otimes g^{-1}\right)
$$

is in $\frac{\Omega}{2}+\left(\Lambda^{2} \mathfrak{l} \oplus \Lambda^{2} \mathfrak{m}\right)$ modulo terms of degree $\geq i+1$, and we put $g_{i+1}=g_{i} g$. This allows to define the sequence $g_{i}$ inductively.

It is clear that the sequence $\rho_{0}^{g_{i}}$ converges, in the sense of formal power series, to a dynamical r-matrix $\rho_{1}$ which is gauge-equivalent to $\rho_{0}$. Moreover $\rho_{1}$ takes values in $\frac{\Omega}{2}+\left(\Lambda^{2} \mathfrak{l} \oplus \Lambda^{2} \mathfrak{m}\right)$ by construction. Let us write $\rho_{1}=\rho_{1}^{\mathfrak{l}}+\rho_{1}^{\mathfrak{m}}$ where $\rho_{1}^{\mathfrak{l}}$ and $\rho_{1}^{\mathfrak{m}}$ take values in $\mathfrak{l} \otimes \mathfrak{l}$ and $\mathfrak{m} \otimes \mathfrak{m}$ respectively. Observe that $\rho_{1}^{\mathfrak{l}}: D \rightarrow \frac{\Omega_{\mathfrak{l}}}{2}+\Lambda^{2} \mathfrak{l}$ is itself a dynamical r-matrix. Hence by Corollary 3.2 we can perform a gaugetransformation for $\mathfrak{l}$ to reduce it to $r_{A M}^{\mathfrak{l}}$.

The following theorem is a generalization to the nonabelian case of [S], Theorem 3 , and will be proved in the next section. 
Theorem 3. Let $r_{0} \in \mathcal{M}_{\Omega}$. Then there exists a unique dynamical $r$-matrix $r=r_{A M}^{\mathfrak{l}}+\frac{\Omega_{\mathfrak{m}}}{2}+t$ with $t: D \rightarrow \Lambda^{2} \mathfrak{m}$, such that $r(0)=r_{0}$.

Corollary 4.3. Under conditions i) and ii) the moduli space $\mathcal{M}(\mathfrak{g}, \mathfrak{l}, \Omega)$ of gaugeequivalence classes of dynamical r-matrices is isomorphic to $\mathcal{M}_{\Omega}$.

Remark 5. We use this opportunity to correct the statement of Theorem 3 in [S] which is false as stated. The mistake is in the proof of Lemma 1, which uses the incorrect statement (4.2). However, the theorem and its proof are correct if one makes in addition the assumption i). In this case it is a special case of Theorem 3 above. Moreover the genericity assumption made in [S] Theorem 3 is not necessary, as the flow constructed in [S] Lemma 2 is well-defined on the whole $\left(\Lambda^{2} \mathfrak{m}\right)^{\mathfrak{l}}$.

Remark 6. Let us identify $\mathfrak{m}$ with $\mathfrak{g} / \mathfrak{l}$ via the decomposition $\mathfrak{g}=\mathfrak{l} \oplus \mathfrak{m}$. This allows to define an action of $G^{\mathfrak{l}}$ on $\mathfrak{m}$, hence also an action of $G^{\mathfrak{l}}$ on $\mathcal{M}_{\Omega}$. It is clear from (3.1) that the isomorphism $\mathcal{M}(\mathfrak{g}, \mathfrak{l}, \Omega) \simeq \mathcal{M}_{\Omega}$ is $G^{\mathfrak{l}}$-equivariant. In particular, $\overline{\mathcal{M}}(\mathfrak{g}, \mathfrak{l}, \Omega) \simeq \mathcal{M}_{\Omega} / G^{\mathfrak{l}}$.

\section{Proof of Theorem 3}

Proof of Theorem 3. We will construct by induction a formal power series $t=$ $\sum_{k} t_{k}$ with $t_{k}: D \rightarrow \Lambda^{2} \mathfrak{m}$ of degree $k$, such that $r=r_{A M}^{\mathfrak{l}}+\frac{\Omega_{\mathfrak{m}}}{2}+t$ is a dynamical r-matrix satisfying $r(0)=r_{0}$. Set $t_{0}=r_{0}-\frac{\Omega}{2} \in \Lambda^{2} \mathfrak{m}$ and let us suppose that we have defined an l-equivariant polynomial $t_{<k}=\sum_{l<k} t_{l}$. Set $s=r_{A M}^{\mathfrak{l}}-\frac{\Omega_{\mathrm{l}}}{2}$, $Z_{\Omega}=C Y B(\Omega)$ and $Z_{\Omega_{\mathfrak{r}}}=C Y B\left(\Omega_{\mathfrak{l}}\right)$. Then the CDYBE for $r_{A M}^{\mathfrak{l}}$ is equivalent to the following equation for $s$ :

$$
\text { Alt }(\overline{d s})+C Y B(s)+\frac{1}{4} Z_{\Omega_{\mathfrak{r}}}=0 .
$$

Let $\pi: \mathfrak{g} \rightarrow \mathfrak{l}$ be the projection along $\mathfrak{m}$. Consider, for $l \leq k$ the following system of differential equations for $i=1, \ldots r$.

$$
\frac{\partial t_{l}}{\partial x_{i}^{*}}=-\left(x_{i}^{*} \otimes 1 \otimes 1\right)\left[\left[t_{<l}^{12}, t_{<l}^{13}\right]+\left[s^{12}+s^{13}, t_{<l}\right]+\frac{1}{4}\left(Z_{\Omega}-Z_{\Omega_{l}}\right)\right]_{l-1}
$$

where by definition $x^{*}(y)=x^{*}(\pi(y))$ for all $x^{*} \in \mathfrak{l}^{*}, y \in \mathfrak{g}$.

Lemma 5.1. Suppose that $\left(E_{l}\right)$ is satisfied for all $l<k$. Then $\left(E_{k}\right)$ admits a unique solution $t_{k}$ of degree $k$, which is $\mathfrak{l}$-equivariant.

Proof. By the equivariant Poincaré lemma, it is enough to show that

$$
\begin{aligned}
& \frac{\partial}{\partial x_{j}^{*}}\left(x_{i}^{*} \otimes 1 \otimes 1\right)\left\{\left[t_{<k}^{12}, t_{<k}^{13}\right]+\left[s^{12}+s^{13}, t_{<k}\right]+\frac{1}{4}\left(Z_{\Omega}-Z_{\Omega_{\mathfrak{r}}}\right)\right\} \\
= & \frac{\partial}{\partial x_{i}^{*}}\left(x_{j}^{*} \otimes 1 \otimes 1\right)\left\{\left[t_{<k}^{12}, t_{<k}^{13}\right]+\left[s^{12}+s^{13}, t_{<k}\right]+\frac{1}{4}\left(Z_{\Omega}-Z_{\Omega_{\mathfrak{r}}}\right)\right\} .
\end{aligned}
$$


Let us write $\partial_{i}$ for $\frac{\partial}{\partial x_{i}^{*}}$ and $t$ for $t_{<k}$. All equations below will be understood modulo terms of degree $\geq k$. Let $X_{i}$ and $X_{j}$ denote the r.h.s and l.h.s of (5.2). Using the assumption that $t$ is a solution of the system $\left(E_{l}\right)$ for all $l<k$, we have

$$
\begin{aligned}
X_{i} & -X_{j} \\
= & \left(x_{i}^{*} \otimes x_{j}^{*} \otimes 1 \otimes 1\right)\left\{\left[\left[t^{12}, t^{13}\right]+\left[s^{12}+s^{13}, t^{23}\right]+\frac{1}{4}\left(Z_{\Omega}-Z_{\Omega_{\mathrm{l}}}\right)^{123}, t^{24}\right]\right. \\
& +\left[t^{23},\left[t^{12}, t^{14}\right]+\left[s^{12}+s^{14}, t^{24}\right]+\frac{1}{4}\left(Z_{\Omega}-Z_{\Omega_{\mathrm{l}}}\right)^{124}\right] \\
& +\left[s^{23}+s^{24},\left[t^{13}, t^{14}\right]+\left[s^{13}+s^{14}, t^{34}\right]+\frac{1}{4}\left(Z_{\Omega}-Z_{\Omega_{\mathrm{l}}}\right)^{134}\right] \\
& -\left[\partial_{i}\left(s^{23}+s^{24}\right), t^{34}\right]+\left[\partial_{j}\left(s^{13}+s^{14}\right), t^{34}\right] \\
& -\left[-\left[t^{12}, t^{23}\right]+\left[-s^{12}+s^{23}, t^{13}\right]-\frac{1}{4}\left(Z_{\Omega}-Z_{\Omega_{\mathrm{\iota}}}\right)^{123}, t^{14}\right] \\
& -\left[t^{13},-\left[t^{12}, t^{24}\right]+\left[-s^{12}+s^{24}, t^{14}\right]-\frac{1}{4}\left(Z_{\Omega}-Z_{\Omega_{\mathrm{l}}}\right)^{124}\right] \\
& \left.-\left[s^{13}+s^{14},\left[t^{23}, t^{24}\right]+\left[s^{23}+s^{24}, t^{34}\right]+\frac{1}{4}\left(Z_{\Omega}-Z_{\Omega_{\mathrm{\iota}}}\right)^{234}\right]\right\} .
\end{aligned}
$$

By the Jacobi identity we have

$$
\left[\left[t^{12}, t^{13}\right], t^{24}\right]+\left[t^{13},\left[t^{12}, t^{24}\right]\right]=\left[\left[t^{12}, t^{23}\right], t^{14}\right]+\left[t^{23},\left[t^{12}, t^{14}\right]\right]=0 .
$$

Moreover,

$$
\begin{aligned}
\left(x_{i}^{*} \otimes x_{j}^{*} \otimes 1 \otimes 1\right)\left\{\left[\frac{1}{4}\left(Z_{\Omega_{\mathfrak{l}}}\right)^{123}, t^{24}\right]+\left[t^{23}, \frac{1}{4}\left(Z_{\Omega_{\mathfrak{l}}}\right)^{124}\right]\right. & \\
+ & {\left.\left[\frac{1}{4}\left(Z_{\Omega_{\mathrm{l}}}\right)^{123}, t^{14}\right]+\left[t^{13}, \frac{1}{4}\left(Z_{\Omega_{\mathrm{l}}}\right)^{124}\right]\right\}=0 }
\end{aligned}
$$

since $Z_{\Omega_{\mathfrak{l}}} \in \Lambda^{3} \mathfrak{l}, t \in \Lambda^{2} \mathfrak{m}$ and $\mathfrak{m}$ is l-invariant. Furthermore, $Z_{\Omega}$ is $\mathfrak{g}$-invariant, hence

$$
\begin{gathered}
\left(x_{i}^{*} \otimes x_{j}^{*} \otimes 1 \otimes 1\right)\left\{\left[\frac{1}{4}\left(Z_{\Omega}\right)^{123}, t^{24}\right]+\left[\frac{1}{4}\left(Z_{\Omega}\right)^{123}, t^{14}\right]\right. \\
\left.+\left[t^{23}, \frac{1}{4}\left(Z_{\Omega}\right)^{124}\right]+\left[t^{13}, \frac{1}{4}\left(Z_{\Omega}\right)^{124}\right]\right\} \\
=\left(x_{i}^{*} \otimes x_{j}^{*} \otimes 1 \otimes 1\right)\left\{-\left[\frac{1}{4}\left(Z_{\Omega}\right)^{123}, t^{34}\right]+\left[t^{34}, \frac{1}{4}\left(Z_{\Omega}\right)^{124}\right]\right\} \\
=-\left(x_{i}^{*} \otimes x_{j}^{*} \otimes 1 \otimes 1\right)\left\{\left[\frac{1}{4}\left(\left(Z_{\Omega_{\mathrm{r}}}\right)^{123}+\left(Z_{\Omega_{\mathrm{r}}}\right)^{124}\right), t^{34}\right]\right\} .
\end{gathered}
$$


In a similar way, $Z_{\Omega}$ and $Z_{\Omega_{1}}$ are l-invariant, hence

$$
\begin{aligned}
{\left[s^{23}+s^{24}, \frac{1}{4}\left(Z_{\Omega}-Z_{\Omega_{\mathrm{r}}}\right)^{134}\right] } & =\left[s^{12}, \frac{1}{4}\left(Z_{\Omega}-Z_{\Omega_{\mathrm{l}}}\right)^{134}\right], \\
-\left[s^{13}+s^{14}, \frac{1}{4}\left(Z_{\Omega}-Z_{\Omega_{\mathrm{r}}}\right)^{234}\right] & =\left[s^{12}, \frac{1}{4}\left(Z_{\Omega}-Z_{\Omega_{\mathrm{l}}}\right)^{234}\right] .
\end{aligned}
$$

From the Jacobi identity again we deduce

$$
\begin{aligned}
& {\left[\left[s^{12}, t^{23}\right], t^{24}\right]+\left[t^{23},\left[s^{12}, t^{24}\right]\right]=\left[s^{12},\left[t^{23}, t^{24}\right]\right],} \\
& {\left[\left[s^{12}, t^{13}\right], t^{14}\right]+\left[t^{13},\left[s^{12}, t^{14}\right]\right]=\left[s^{12},\left[t^{13}, t^{14}\right]\right],} \\
& {\left[\left[s^{13}, t^{23}\right], t^{24}\right]-\left[s^{13},\left[t^{23}, t^{24}\right]\right]=0,} \\
& {\left[s^{24},\left[t^{13}, t^{14}\right]\right]-\left[t^{13},\left[s^{24}, t^{14}\right]\right]=0,} \\
& {\left[t^{23},\left[s^{14}, t^{24}\right]\right]-\left[s^{14},\left[t^{23}, t^{24}\right]\right]=0,} \\
& \left.\left[s^{23},\left[t^{13}, t^{14}\right]\right]-\left[s^{23}, t^{13}\right], t^{14}\right]=0 .
\end{aligned}
$$

and

$$
\begin{aligned}
{\left[s^{23}+s^{24}\right.} & \left.,\left[s^{13}+s^{14}, t^{34}\right]\right]-\left[s^{13}+s^{14},\left[s^{23}+s^{24}, t^{34}\right]\right] \\
& =\left[\left[s^{23}, s^{13}\right], t^{34}\right]+\left[\left[s^{24}, s^{14}\right], t^{34}\right] \\
& =-\left[\left[s^{13}, s^{23}\right], t^{34}\right]-\left[\left[s^{14}, s^{24}\right], t^{34}\right] .
\end{aligned}
$$

Collecting terms from $(5.4),(5.5),(5.6),(5.7),(5.8),(5.9)$ and replacing in (5.3), we obtain

$$
\begin{aligned}
X_{i}-X_{j}= & \left(x_{i}^{*} \otimes x_{j}^{*} \otimes 1 \otimes 1\right)\left\{-\left[\frac{1}{4}\left(\left(Z_{\Omega_{\mathrm{f}}}\right)^{123}+\left(Z_{\Omega_{\mathrm{l}}}\right)^{124}\right), t^{34}\right]\right. \\
& -\left[\left[s^{13}, s^{23}\right], t^{34}\right]-\left[\left[s^{14}, s^{24}\right], t^{34}\right]-\left[\partial_{i}\left(s^{23}+s^{24}\right), t^{34}\right] \\
& +\left[\partial_{j}\left(s^{13}+s^{14}\right), t^{34}\right]+\left[s^{12},\left[t^{23}, t^{24}\right]+\frac{1}{4}\left(Z_{\Omega}-Z_{\Omega_{\mathrm{l}}}\right)^{234}\right] \\
& \left.+\left[s^{12},\left[t^{13}, t^{14}\right]+\frac{1}{4}\left(Z_{\Omega}-Z_{\Omega_{\mathrm{l}}}\right)^{134}\right]\right\}
\end{aligned}
$$

Using the fact that $t$ is a solution to the system $\left(E_{l}\right)$ again we have

$$
\begin{aligned}
& {\left[s^{12},\left[t^{23}, t^{24}\right]+\frac{1}{4}\left(Z_{\Omega}-Z_{\Omega_{\mathrm{r}}}\right)^{234}\right]+\left[s^{12},\left[t^{13}, t^{14}\right]+\frac{1}{4}\left(Z_{\Omega}-Z_{\Omega_{\mathrm{r}}}\right)^{134}\right]} \\
& =\left[s^{12}, \sum_{k}\left(x_{k} \otimes 1+1 \otimes x_{k}\right) \otimes\left(-\partial_{k} t-\left[s_{k} \otimes 1+1 \otimes s_{k}, t\right]\right)\right] \\
& =-\left[s^{12}, \sum_{k}\left(x_{k} \otimes 1+1 \otimes x_{k}\right) \otimes \partial_{k} t\right]-\left[s^{12},\left[s^{23}+s^{24}, t^{34}\right]\right] \\
& -\left[s^{12},\left[s^{13}+s^{14}, t^{34}\right]\right]
\end{aligned}
$$

where we set $s_{k}=\left(x_{k}^{*} \otimes 1\right) s$. But $s$ is $\mathfrak{l}$-equivariant, i.e for $y \in \mathfrak{l}$ we have

$$
[s, y \otimes 1+1 \otimes y]=\sum_{l}\left[x_{l}, y\right] \partial_{l} s
$$


where $\left[x_{l}, y\right]$ is considered as a function $D \rightarrow \mathbb{C}$. Thus,

$$
\begin{aligned}
-\left[s^{12}, \sum_{k}\left(x_{k} \otimes 1+1 \otimes x_{k}\right) \otimes \partial_{k} t\right] & =-\sum_{l, k} \partial_{l} s^{12} \partial_{k} t^{34}\left[x_{l}, x_{k}\right] \\
& =-\sum_{l} \partial_{l} s^{12}\left[x_{l}^{3}+x_{l}^{4}, t^{34}\right] .
\end{aligned}
$$

Using Jacobi identity, we can write

$$
\begin{aligned}
& {\left[s^{12},\left[s^{23}+s^{24}, t^{34}\right]\right]=\left[\left[s^{12}, s^{23}\right], t^{34}\right]+\left[\left[s^{12}, s^{24}\right], t^{34}\right],} \\
& {\left[s^{12},\left[s^{23}+s^{24}, t^{34}\right]\right]=\left[\left[s^{12}, s^{13}\right], t^{34}\right]+\left[\left[s^{12}, s^{14}\right], t^{34}\right] .}
\end{aligned}
$$

Using (5.10), (5.11), (5.12) and (5.13) we finally get, by (5.1)

$$
\begin{aligned}
X_{i}-X_{j}= & -\left(x_{i}^{*} \otimes x_{j}^{*} \otimes 1 \otimes 1\right) \cdot \\
& \left\{\left[\operatorname{Alt}(\overline{d s})^{123}+\left[s^{13}, s^{23}\right]+\left[s^{12}, s^{13}\right]+\left[s^{12}, s^{23}\right]+\frac{1}{4}\left(Z_{\Omega_{\mathrm{r}}}\right)^{123}, t^{34}\right]\right. \\
& \left.+\left[\operatorname{Alt}(\overline{d s})^{124}+\left[s^{14}, s^{24}\right]+\left[s^{12}, s^{14}\right]+\left[s^{12}, s^{24}\right]+\frac{1}{4}\left(Z_{\Omega_{\mathrm{f}}}\right)^{124}, t^{34}\right]\right\} \\
= & 0
\end{aligned}
$$

Let $t=\sum t_{i}: D \rightarrow \Lambda^{2} \mathfrak{m}$ be the l-equivariant series constructed by applying Lemma 4.2 succesively, starting from $t_{0}$.

Consider the algebraic variety

$$
\mathcal{T}_{\Omega}=\left\{t \in \Lambda^{2} \mathfrak{m} \mid C Y B\left(t+\frac{\Omega}{2}\right)=0 \text { in } \Lambda^{3}(\mathfrak{g} / \mathfrak{l})\right\} .
$$

Let $x^{*} \in \mathfrak{l}^{*}$ and consider the flow on $\Lambda^{2} \mathfrak{m}$ defined by the equation

$$
\begin{aligned}
& \frac{\partial u}{\partial \epsilon}= \\
& -\left(x^{*} \otimes 1 \otimes 1\right)\left(\left[u^{12}, u^{13}\right]+\left[s^{12}+s^{13}, u^{23}\right]+\frac{1}{4}\left(C Y B(\Omega)-C Y B\left(\Omega_{\mathfrak{l}}\right)\right)\right) .
\end{aligned}
$$

Lemma 5.2. The flow (5.14) preserves $\mathcal{T}_{\Omega}$.

Proof. Let $u \in \mathcal{T}_{\Omega}$. Set $h_{1}=\left(x^{*} \otimes 1 \otimes 1\right)\left(\left[s^{12}+s^{13}, u^{23}\right]\right)$,

$$
h_{2}=\left(x^{*} \otimes 1 \otimes 1\right)\left(\left[u^{12}, u^{13}\right]+\frac{1}{4}\left(Z_{\Omega}-Z_{\Omega_{\mathrm{l}}}\right)\right) \text {. }
$$

Note that $h_{1} \in \Lambda^{2} \mathfrak{m}$ by condition i) and that $h_{2} \in \Lambda^{2} \mathfrak{m}$ since $u \in \Lambda^{2} \mathfrak{m}$ and since by ii),

$$
Z_{\Omega}-Z_{\Omega_{\mathfrak{l}}} \in(\mathfrak{m} \otimes \mathfrak{g} \otimes \mathfrak{g}) \oplus(\mathfrak{l} \otimes \mathfrak{m} \otimes \mathfrak{m}) .
$$

It thus remains to check that the vector field defined by (5.14) is tangent to $\mathcal{T}_{\Omega}$, i.e that $C Y B\left(u, h_{1}+h_{2}\right) \in$ Alt $(\mathfrak{l} \otimes \mathfrak{g} \otimes \mathfrak{g})$, where we use the notation

$$
C Y B(a, b)=\left[a^{12}, b^{13}\right]+\left[a^{13}, b^{23}\right]+\left[a^{12}, b^{23}\right]+\left[b^{12}, a^{13}\right]+\left[b^{13}, a^{23}\right]+\left[b^{12}, a^{23}\right] \text {. }
$$


But

$$
C Y B\left(u, h_{1}\right)=\operatorname{ad}\left(\left(x^{*} \otimes 1\right) s\right) C Y B(u) \in \operatorname{Alt}(\mathfrak{l} \otimes \mathfrak{g} \otimes \mathfrak{g}),
$$

and $C Y B\left(u, h_{2}\right) \in$ Alt $(\mathfrak{l} \otimes \mathfrak{g} \otimes \mathfrak{g})$ by $[\mathrm{S}]$, Lemma 3 (note that the commutativity of $\mathfrak{l}$, assumed in $[\mathrm{S}]$, is not used in the proof of Lemma 3$)$.

Corollary 5.1. The map $t: D \rightarrow \Lambda^{2} \mathfrak{m}$ takes values in $\mathcal{T}_{\Omega}$.

Proof. Note that $t(0) \in \mathcal{T}_{\Omega}$ by assumption, and that for any $x^{*} \in D$ the function $u(\epsilon)=t\left(\epsilon x^{*}\right)$ on the formal disc satisfies (5.14) by construction. Hence $t$ takes values in $\mathcal{T}_{\Omega}$.

We now conclude the proof of Theorem 3 by showing that $r=r_{A M}^{\mathfrak{l}}+\frac{\Omega_{\mathfrak{m}}}{2}+t$ is a dynamical r-matrix. Setting $s_{A M}^{\mathfrak{l}}=r_{A M}^{\mathfrak{l}}-\frac{\Omega_{\mathrm{l}}}{2}$ we have

$$
\begin{aligned}
\operatorname{Alt}(\overline{d r})+C Y B(r) \\
=\operatorname{Alt}\left(\overline{d s_{A M}^{\mathfrak{l}}}\right)+\operatorname{Alt}(\overline{d t})+C Y B\left(s_{A M}^{\mathfrak{l}}+t\right)+\frac{1}{4} C Y B(\Omega) \\
=\operatorname{Alt}\left(\overline{d s_{A M}^{\mathfrak{l}}}\right)+\operatorname{Alt}(\overline{d t})+C Y B\left(s_{A M}^{\mathfrak{l}}\right)+C Y B(t)+C Y B\left(s_{A M}^{\mathfrak{l}}, t\right) \\
\quad+\frac{1}{4} C Y B(\Omega) .
\end{aligned}
$$

Using the CDYBE for $r_{A M}^{\mathfrak{l}}$ we see that $r$ is a dynamical r-matrix if and only if

$$
\text { Alt }(\overline{d t})+C Y B(t)+C Y B\left(s_{A M}^{\mathfrak{l}}, t\right)+\frac{1}{4}\left(C Y B(\Omega)-C Y B\left(\Omega_{\mathfrak{l}}\right)\right)=0 .
$$

Since $t$ takes values in $\mathcal{T}_{\Omega},(5.15)$ is equivalent to the system

$$
\frac{\partial t}{\partial x_{i}^{*}}=-\left(x_{i}^{*} \otimes 1 \otimes 1\right)\left(C Y B(t)+C Y B\left(s_{A M}^{\mathfrak{l}}, t\right)+\frac{1}{4}\left(C Y B(\Omega)-C Y B\left(\Omega_{\mathfrak{l}}\right)\right)\right)
$$

for $i=1, \ldots r$. It is easy to see from conditions i) and ii) that this last system is itself equivalent to the collection of systems $\left(E_{l}\right)$ for all $l \in \mathbb{N}$.

\section{Appendix. Generalized Alekseev-Meinrenken dynamical r-matrices}

In this appendix we give a generalization of the dynamical r-matrix $r_{A M}^{\mathfrak{l}}$.

Let $\mathfrak{g}$ be a finite-dimensional complex Lie algebra and $B: \mathfrak{g} \rightarrow \mathfrak{g}$ an automorphism of order $n$. Then $\mathfrak{g}=\bigoplus_{j \in \mathbb{Z} / n \mathbb{Z}} \mathfrak{g}_{j}$ where $\mathfrak{g}_{j}=\operatorname{Ker}\left(B-e^{\frac{2 i \pi j}{n}}\right)$. Set $\mathfrak{l}=\mathfrak{g}_{0}$. Then $\mathfrak{g}_{0}$ acts on $\mathfrak{g}_{j}$ for all $j$.

Assume that $\mathfrak{g}$ carries a nondegenerate invariant form $($,$) , which is stable$ under $B$. Set $\Omega=(,)^{-1} \in\left(S^{2} \mathfrak{g}\right)^{\mathfrak{g}}$. We will identify $\mathfrak{g}$ with $\mathfrak{g}^{*}$ and $\mathfrak{l}$ with $\mathfrak{l}^{*}$ using $($,$) .$

Let $D$ be the formal neighborhood of zero in $\mathfrak{l}^{*} \simeq \mathfrak{l}$. Consider the function $\hat{\rho}: D \rightarrow \operatorname{End}(\mathfrak{g})$ such that $\hat{\rho}(A)_{\mid \mathfrak{g}_{i}}=f_{i}(\operatorname{ad} A)$, with

$$
\begin{aligned}
& f_{0}(s)=\frac{1}{s}-\frac{1}{2} \operatorname{cotanh}\left(\frac{1}{2} s\right), \\
& f_{j}(s)=-\frac{1}{2} \operatorname{cotanh}\left(\frac{1}{2}\left(s+\frac{2 i \pi j}{n}\right)\right), \quad j \neq 0 .
\end{aligned}
$$


The element $\hat{\rho}$ defines a map $\rho: D \rightarrow \Lambda^{2} \mathfrak{g}$. Let us set $r_{B}=\frac{\Omega}{2}+\rho$.

Theorem A 1. The map $r_{B}$ is a dynamical r-matrix.

Remark 7. If $B=1$ then $r_{B}$ is equal to the Alekseev-Meinrenken dynamical r-matrix $r_{A M}^{\mathfrak{l}}$.

The rest of this appendix is devoted to the proof of Theorem A.1. We start by recalling the following result from $[\mathrm{EV}]$. Let $\mathfrak{l}$ be a reductive Lie algebra with Cartan subalgebra $\mathfrak{h}, \mathfrak{g}$ any finite-dimensional Lie algebra containing $\mathfrak{l}$ and let $\Omega \in\left(S^{2} \mathfrak{g}\right)^{\mathfrak{g}}$. The projection $\mathfrak{l} \rightarrow \mathfrak{h}$ defines an embedding $\mathfrak{h}^{*} \rightarrow \mathfrak{l}^{*}$. Let $\Delta$ be the root system of $\mathfrak{l}$ and $\mathfrak{l}_{\alpha}$ the weight subspace corresponding to $\alpha \in \Delta$. Choose an nondegenerate invariant inner product on $\mathfrak{l}$. Let us fix $e_{\alpha} \in \mathfrak{l}_{\alpha}$ for all $\alpha \in \Delta$ such that $\left(e_{-\alpha}, e_{\alpha}\right)=1$. Define a function $\rho_{0}: D \rightarrow \Lambda^{2} \mathfrak{l} \subset \Lambda^{2} \mathfrak{g}$ by

$$
\rho_{0}(\lambda)=\sum_{\alpha>0} \frac{e_{\alpha} \otimes e_{-\alpha}-e_{\alpha} \otimes e_{-\alpha}}{(\alpha, \lambda)} .
$$

It is clear that $\rho_{0}$ does not depend on the choice of the inner product.

Let $r: \mathfrak{l}^{*} \rightarrow \mathfrak{g} \otimes \mathfrak{g}$ be an $\mathfrak{l}$-equivariant meromorphic function satisfying the quasi-unitarity condition $r+r^{21}=\Omega$.

Theorem A 2 ([EV], Theorem 3.14). The map $r$ is a classical dynamical $r$ matrix if and only if $r_{\mid \mathfrak{h}^{*}}+\rho_{0}$ is a classical dynamical r-matrix for $\mathfrak{h}$.

Proof. This is proved in $[\mathrm{EV}]$ under the assumption that $\mathfrak{g}$ is simple and $\mathfrak{h} \subset \mathfrak{g}$ is a Cartan subalgebra. However, this assumption is not used in the proof and the result is valid in general.

Proposition A 1. Theorem A1 is valid if $\mathfrak{l}$ is reductive, $\mathfrak{g}=\mathfrak{l}_{1} \oplus \cdots \oplus \mathfrak{l}_{n}$ with $\mathfrak{l}_{i}=\mathfrak{l}$, and $B$ is the cyclic permutation automorphism $B: \mathfrak{l}_{i} \stackrel{\sim}{\rightarrow} \mathfrak{l}_{i+1} \bmod n$.

Proof. Let $\left(x_{i}\right)_{i \in I}$ be an orthonormal basis of $\mathfrak{h} \subset \mathfrak{l}$. For $i=1, \ldots, n$ we will write $e_{\alpha}^{(i)}$ for the element of $\mathfrak{l}_{i} \subset \mathfrak{g}$ corresponding to $e_{\alpha}$. With this notation, we have $B\left(e_{\alpha}^{(i)}\right)=e_{\alpha}^{(i+1)}$ and

$$
\mathfrak{g}_{j}=\left\{g_{1}^{(1)} \oplus \cdots \oplus g_{n}^{(n)} ; \mid g_{k+1}=e^{\frac{-2 i \pi j}{n}} g_{k}\right\} .
$$

Finally, let $\mathfrak{k} \subset \bigoplus_{i} \mathfrak{h}^{(i)}$ be the orthogonal complement to $\mathfrak{h}$. Note that $1-B$ restricts to an invertible operator on $\mathfrak{k}$. A direct computation shows that

$$
\begin{aligned}
& r_{B \mid \mathfrak{h}^{*}}+\rho_{0} \\
& =\frac{\Omega}{2}+\sum_{\alpha>0, i} e_{\alpha}^{(i)} \wedge\left(-\frac{1}{2}\left(\frac{1+B e^{(\alpha, \lambda)}}{1-B e^{(\alpha, \lambda)}}\right) e_{-\alpha}^{(i)}\right)+\sum_{i} y_{i} \otimes\left(-\frac{1+B}{2(1-B)} y_{i}\right) \\
& =\sum_{i} x_{i} \otimes x_{i}+\sum_{\alpha>0, i} e_{-\alpha}^{(i)} \otimes e_{\alpha}^{(i)}-\sum_{\alpha>0, i} \sum_{l \geq 1} e^{l(\alpha, \lambda)} e_{\alpha}^{(i)} \wedge e_{-\alpha}^{(i+l)} \\
& +\frac{1}{2} \sum_{i} \frac{B+1}{B-1} y_{i} \otimes y_{i}
\end{aligned}
$$


where $\left(y_{i}\right)_{i \in J}$ is an orthonormal basis of $\mathfrak{k}$. By [S], Theorem 4 this expression is a dymamical r-matrix. Hence, by Theorem A.2, $r_{B}$ is a dynamical r-matrix.

Define a map $W: D \rightarrow \Lambda^{3} \mathfrak{g}$ by

$$
W(A)=\operatorname{Alt}(\overline{d \rho(A)})+C Y B(\rho(A))+\frac{1}{4} Z
$$

where $Z=C Y B(\Omega)$. For any $i, j \in \mathbb{Z} / n \mathbb{Z}, X \in \mathfrak{g}_{i}, Y \in \mathfrak{g}_{j}$ and $A \in \mathfrak{l}$, consider the expression

$$
K_{i j}(A, X, Y)=(1 \otimes X \otimes Y, W(A)) \in \mathfrak{g}_{i+j} .
$$

Lemma A 1. The expression $K_{i j}(A, X, Y)$ is given by a universal Lie series in $A, X$ and $Y$.

Proof. Straightforward.

Moreover, from Proposition A.1 we deduce the following result.

Proposition A 2. We have $K_{i j}(A, X, Y)=0$ for all $A, X, Y$ if $\mathfrak{l}=\mathfrak{g l}_{n}(\mathbb{C})$, $\mathfrak{g}=\mathfrak{l}_{1} \oplus \cdots \oplus \mathfrak{l}_{n}$ with $\mathfrak{l}_{k}=\mathfrak{l}$, and $B$ is the cyclic permutation automorphism.

Finally, we recall the following standard fact.

Lemma A 2. Let $P\left(X_{1}, \ldots, X_{n}\right)$ be a Lie polynomial which vanishes identically for all $X_{1}, \ldots X_{n} \in \mathfrak{g l}_{k}(\mathbb{C})$ for all $k \in \mathbb{N}$. Then $P\left(X_{1}, \ldots, X_{n}\right)=0$.

Proof. Let $F_{n}$ be the free Lie algebra in $n$ generators and let $U_{n}$ be its enveloping algebra (the free associative algebra). Let $d$ be the degree of $P$ and let $I$ be the ideal in $U_{n}$ generated by elements of degree at least $d+1$. Then $U_{n} / I$ is a finitedimensional algebra. Let $\sigma: U_{n} / I \rightarrow \mathfrak{g l}\left(U_{n} / I\right)$ be the left regular representation. Then $\sigma\left(P\left(X_{1}, \ldots, X_{n}\right)\right)=0$. Hence $P\left(X_{1}, \ldots, X_{n}\right)=0$.

Now, let us write $K_{i j}=\sum_{k} K_{i j}^{(k)}$ where $K_{i j}^{(k)}$ is the homogeneous component of degree $k$. By Proposition A.2, $K_{i j}^{(k)}(A, X, Y)=0$ whenever $A, X, Y \in \mathfrak{g l}_{m}(\mathbb{C})$ for some $m \in \mathbb{C}$. Hence $K_{i j}^{(k)}=0$ by Lemma A.2. Thus $W(A)=0$ for all $A \in \mathfrak{l}$. Theorem A.1 is proved.

Examples. Let $\mathfrak{g}$ be a simple complex Lie algebra and $\mathfrak{l} \subset \mathfrak{g}$ a semisimple subalgebra with same rank as $\mathfrak{g}$. Such pairs are classified in [BdS]. Let $Q_{\mathfrak{r}}$ and $Q_{\mathfrak{g}}$ be the root lattices of $\mathfrak{l}$ and $\mathfrak{g}$ respectively and set $\Gamma=Q_{\mathfrak{g}} / Q_{\mathfrak{r}}$. It follows from $[\mathrm{BdS}]$ that $\Gamma$ is one of the groups $\mathbb{Z} / 2 \mathbb{Z}, \mathbb{Z} / 3 \mathbb{Z}$ or $\mathbb{Z} / 5 \mathbb{Z}$ (the case $\Gamma=\mathbb{Z} / 2 \mathbb{Z}$ corresponds to symmetric spaces). Let $\chi$ be a nontrivial character $\chi$ of $\Gamma$. Then $\chi$ gives rise to an automorphism $B_{\chi}$ of $\mathfrak{g}$ whose set of fixed points is $\mathfrak{l}$, defined by

$$
B_{\chi \mid \mathfrak{g}_{\alpha}}=\chi(\alpha) I d
$$


where $\mathfrak{g}_{\alpha}$ is the root space of weight $\alpha$. Let $\Omega \in\left(S^{2} \mathfrak{g}\right)^{\mathfrak{g}}$ be a Casimir element $(\Omega \neq 0)$. Let $r: D \rightarrow \mathfrak{g} \otimes \mathfrak{g}$ be a dynamical $\mathrm{r}$-matrix such that $r+r^{21}=\Omega$. It follows from Theorem A.2 and [EV] Theorem 3.1, that, up to gauge-equivalence,

$$
r_{\mid \mathfrak{h}^{*}}+\rho_{0}=\frac{\Omega}{2}+\sum_{\alpha \in \Delta_{\mathfrak{g}}} \frac{1}{2} \operatorname{cotanh}\left(\frac{1}{2}(\alpha, \lambda-\nu)\right) e_{\alpha} \otimes e_{-\alpha},
$$

for some $\nu \in \mathfrak{h}^{*}$, where $\Delta_{\mathfrak{g}}$ is the root system of $\mathfrak{g}$. But then $r$ is regular at $\lambda=0$ if and only if $(\alpha, \nu)=0$ modulo $2 \pi i \mathbb{Z}$ for all $\alpha \in \Delta_{\mathfrak{r}}$ and $(\alpha, \nu) \neq 0$ modulo $2 \pi i \mathbb{Z}$ for all $\alpha \in \Delta_{\mathfrak{g}} \backslash \Delta_{\mathfrak{l}}$, where $\Delta_{\mathfrak{l}} \subset \Delta_{\mathfrak{g}}$ is the root system of $\mathfrak{l}$. Such $\nu$ defines a nontrivial character $\chi$ of $\Gamma$, and it follows from [EV], Section 3.8 that $r$ is gauge-equivalent to the generalized Alekseev-Meinrenken dynamical r-matrix $r_{B_{\chi}}$. Hence the moduli space $\mathcal{M}(\mathfrak{g}, \mathfrak{l}, \Omega)$ consists of $|\Gamma|-1$ points.

\section{Acknowledgments}

The authors are grateful to P. Xu for useful discussions. The first author was supported by the NSF grant DMS-9700477. P.E performed this research as a CMI prize fellow. O.S conducted this research partially for the Clay Mathematics Institute, and thanks the MIT mathematics department for hospitality.

\section{References}

[AM] A. Alekseev and E. Meinrenken, The non-commutative Weil algebra., Invent. Math. 139 (2000), 135-172.

[BdS] A. Borel and J. De Siebenthal, Les sous-groupes fermés de rang maximum des groupes de Lie clos., Comment. Math. Helv. 23 (1949), 200-221.

[ES] P. Etingof and O. Schiffmann, Lectures on dynamical Yang-Baxter equations, to appear in the Proceedings of the Durham conference (1999). math.QA/9908064.

[EV] P. Etingof and A. Varchenko, Geometry and classification of solutions of the classical dynamical Yang-Baxter equation, Comm. Math. Phys. 192 (1998), 77-120 .

[Fe] G. Felder, Conformal field theory and integrable systems associated to elliptic curves, Proceedings of the International Congress of Mathematicians, Zürich 1994, 1247-1255, Birkhäuser, 1995.

[S] O. Schiffmann, On classification of dynamical r-matrices, Math. Res. Lett. 5 (1998), 13-30.

[Xu] P. Xu, Triangular dynamical r-matrices and quantization, preprint, math.QA/0005006.

Dept. of Mathematics, Mit,77 Massachusetts avenue, 02138 CAMBRidGE, MA.

E-mail address: etingof@math.mit.edu

Dept. of Mathematics, Yale, 10-12 Hillhouse Avenue, 06510 NEW HaVen, CT.

E-mail address: schiffmann@gauss.math.yale.edu 